\title{
Assessment of Diversity and Traditional Uses of Bryophytes Along Some Hill Roads in a Biodiversity Hot Spot Region of India-A Case Study of Mizoram
}

\author{
Samar Kumar Banerjee*, Anjani Kumar Srivastava \\ University Department of Botany, Ranchi University, Ranchi, India \\ Email address: \\ samarbanerjeel@gmail.com (S. K. Banerjee), anjani.ranjana@gmail.com (A. K. Srivastava) \\ ${ }^{*}$ Corresponding author
}

To cite this article:

Samar Kumar Banerjee, Anjani Kumar Srivastava. Assessment of Diversity and Traditional Uses of Bryophytes Along Some Hill Roads in a Biodiversity Hot Spot Region of India-A Case Study of Mizoram. International Journal of Natural Resource Ecology and Management. Vol. 4, No. 3, 2019, pp. 73-82. doi: 10.11648/j.ijnrem.20190403.12

Received: May 19, 2019; Accepted: June 24, 2019; Published: July 10, 2019

\begin{abstract}
In India, the bryophytes are represented by 2562 taxa (1636 mosses, 887 liverworts and 39 hornworts). They usually inhabit narrow ecological niches with preference for damp and shady conditions. In Himalayas the bryophytes are rich in diversity and well represented due to prevailing of high rainfall and humidity. Eastern Himalayas are richest in bryophyte flora. Bryophytes are of great ecological importance, they are Pioneer of the land plants and the first plants to grow and colonize the barren rocks and lands. In India, the bryophytes are represented by 2562 taxa (1636 mosses, 887 liverworts and 39 hornworts). Along the Mizoram roads 76 species of bryophytes distributed over 29 families have been identified and recorded by the authors. These bryophytes provide vital ecosystem services like soil formation, habitat modification and nutrient cycling and are useful in pollution detection and monitoring. The flavonoids and terpenoids contained in majority of them show various biological activities with considerable potential of chemical and pharmaceutical properties. Road side village people in Mizoram generally use these bryophytes as medicines, vegetable and for prevention of soil erosion. It has been assessed that during road development these flora will be impacted and that will have impact on community settled along the road. Effort has been made in this paper to assess the diversity of bryophytes along the road corridors so that proper planning can be done during road development to save this natural resource.
\end{abstract}

Keywords: Amphibian, Flavonoids, Terpenoids, Anticancerous, Erosion

\section{Introduction}

Bryophytes, especially the terrestrial liverworts, are among the pioneers of land vegetation on earth and because of their small size have the ability to colonize nearly all conceivable habitats (micro-environments) in small "pockets" or "niches" which sometimes limit the distribution of certain taxa. They are accepted as the oldest living land plants with the exception of a few aquatic species as fossilized spores of early land plants from the mid-Ordovician (approximately 470 million years ago) have been interpreted as having been produced by early liverworts $[1,2]$. Therefore, these plants are considered as an ancient group of plants positioned between Algae and Bryophytes. They normally have a boreal habitat and thrive in humid conditions. As water is indispensible for the act of fertilization they are also known as Amphibians of the Plant Kingdom. In India, the bryophytes are represented by 2562 taxa (1636 mosses, 887 liverworts and 39 hornworts). They usually inhabit narrow ecological niches with preference for damp and shady conditions. In India, Bryophytes are quite abundant in both Nilgiri hills and Himalayas. Eastern Himalayas are the richest in bryophyte flora. The eastern Himalayas exhibited a more luxurious growth of bryophytes compared to the western Himalayas on account of much rainfall and more humidity in the atmosphere [3].

The bryophytes provide vital ecosystem services like soil formation, habitat modification and nutrient cycling and are useful in pollution detection and monitoring. The flavonoids and terpenoids contained in majority of them show various 
biological activities with considerable potential of chemical and pharmaceutical properties [4]. The Bryophytes are not considered to be of much economic value because except a few Bryophytes none of these are of direct use to man. However, they play an important role in the economy of the nature. Bryophytes are also potential sources of new antibiotics and anti-cancerous substances [5]. The recent upsurge of interest in monitoring atmospheric pollution has revealed a new aspect of the relevance of this elegant group of plant kingdom. They have been more rationally used in pollution detection, environmental monitoring and as climatic indicator which can be helpful to management of a particular environment. They act as reservoirs of moisture which provide breeding places for insects, small animals and also excellent propagation beds for plant seeds. They offer microhabitats that are critical to the survival of a tremendous diversity of organisms such as single-celled eukaryotes, protozoa, and numerous groups of invertebrates [6]. These plants are also important as environmental and ecological indicators. In India, initial studies are being conducted to monitor trace element pollution using mosses as biomonitors [7]. Liverworts, in concert with mosses and hornworts, play a significant role in the global carbon budget and $\mathrm{CO}_{2}$ exchange, plant succession, nutrient cycling, and water retention [8-11]. These groups of land plants also have been used as indicators of past climate change, to validate climate models, and as potential indicators of global warming [12, 13].

Mosses constitute the major component of Indian bryoflora with about 2000 species (including intraspecific categories) belonging to 342 genera and 54 families of these, ca. 1030 occur in Eastern Himalaya. Liverworts are represented by ca. 850 species under 140 genera and 52 families [14, 15]. The majority of them are damp loving, predominantly terrestrial and favour shaded conditions, whereas a number of taxa are epiphytic epiphyllous aquatic and xeromorphic. About 260 taxa or ca. $30 \%$ of the total liverwort flora are endemic to different regions of the country with maximum representation in Eastern Himalaya, which account for ca. $60 \%$ of the total endemic taxa in the country [16].

Along the Mizoram roads which are part of Eastern Himalaya, 76 species of bryophytes distributed over 29 families have been identified and recorded by the authors. 65 taxa of Bryophytes distributed under 50 genera and 36 families from Mamit district of Mizoram, of these, the mosses are represented by 37 species of 28 genera and 17 families, while Liverworts are represented by 26 species of 20 genera and 18 families. It has also been reported that 41 taxa of Bryophytes distributed under 34 genera and 23 families have been recorded. Of these, the mosses are represented by 23 species of 20 genera and 14 families, while Liverworts are represented by 15 species of 12 genera and 8 families.

Road side village people in Mizoram generally use these bryophytes as medicines, vegetable and for prevention of soil erosion. It has been assessed that during road development these flora will be impacted and that will have impact on community settled along the road. Effort has been made in this paper to assess the diversity of bryophytes along the road corridors so that proper planning can be done during road development to save this natural resource. 127 species of Mosses under 71 genera belonging to 27 families has been reported from the Assam Valley Wet Evergreen forests, Assam, India [17].

\section{Materials and Methods}

\subsection{Study Area}

For listing of the Bryophytes along the roads, three major roads of Mizoram situated in four different locations were selected, namely - Aizawl- Lunglei road, Sheling-Champhai road, Thenzal-Serchip road and Lunglei -Tlabung road (Figure 1). All the roads were surveyed with team of experts for three consecutive years during the months of October to January (2016 to 2018). Data were collected within 200m (considering corridor of impact) of the roads by traversing on foot along all the Bryophyte habitats on both side of the roads (valley side and hill side). Vegetation water channels, ridges and various habitats within the corridor of impact ( $200 \mathrm{~m}$ of the road) were also considered for the listing of the Bryophytes.

\subsection{Collection of Specimen}

The collected fresh materials were examined and fixed in $70 \%$ alcohol as well as mosses were air dried, pressed and stored in suitable well labeled standard sized paper packets as per the internationally accepted herbarium methodology with proper field notes. All the specimens were collected in fertile stage and Mossess were processed through conventional herbarium [18]. They were identified by matching them with herbarium specimens of local universities and research institutions and also by expert team. The identification of taxa has been done in the laboratory by studying the specimens and consulting with various standard literatures.

\subsection{Taxonomy}

Identification has been done on basis of detailed morphotaxonomic studies of the material under the Binoculars, Dissceting Microscope, Compound Microscope and Trinocular Microscope. The plants were identified using the different floras [19-22]. Other standard literature were also consulted for identification of the bryophyte flora [23-25]. However, the genera and species taxonomic citation is based on published literature and The Plant List.

Status of the listed Bryophytes in IUCN Red list along the roads under study has been assessed as per of the IUCN Red list 2017 version 3.1. Those not finding place in IUCN red list were assessed as per annual Catalogue of life checklist 2017.

The data on the uses of the Bryophytes were collected through questionnaire, interviews and discussions among local people in presence of the Village Council (VCP), local Vadhya and old men and women of local village community along the roads. 


\section{Results and Discussion}

\subsection{Diversity of Bryophytes Along Roads}

For listing of the Bryophytes along the roads, four major roads of Mizoram situated in four different locations were selected, namely - Aizawl -Thenzal Lunglei road, ShelingChamphai road, Thenzal-Serchip road and Lunglei-TlabungKwarpuicchua road were selected for this study (Figure 1).

Total 76 Bryophytes were recorded in four roads distributed over 39 families. Four habitats for Bryophytes were identified along the roads. 72 bryophyte species were recorded from Aizawl -Thenzal Lunglei road, 64 species recorded from Sheling-Champhai road, 67 species were recoded from Thenzal-Serchip and 55 species were recorded from Lunglei-Tlabung-Kwarpuicchua road (Table 1 and Figure $2 \& 3$ ).

These habitats are-Terrestrial, growing on moist surface of road side hills (40-53\%), Terrestrial, growing on road side water logged area (submerged ), Terrestrial, growing on road side tree bark surface (20-26\%), Terrestrial, growing on road side moist rocky surface (16-21\% ). Habitat wise distribution of bryophytes indicate that out of 76 species 40 species
(53\%) found growing on moist surface of road side hills (40$53 \%$ ), 20 species (26\%) found growing on road side tree bark surface and 16 species $(21 \%)$ growing on road side moist rocky surface.(Figure 4 )

Bryophytes collected and identified along the roads are not listed in IUCN Red list Version 3.1 and Catalogue of life annual checklist 2018.

\subsection{Use of Bryophytes by Local People in the Villages Along the Roads}

Many tribal communities and rural populations who are living along the road are dependent upon such natural resources like Bryophytes distributed along the roads for treatment of various ailments and diseases and also as food and fodder supplement. Road side village people in Mizoram generally use these Bryophytes as medicines, vegetable and for prevention of soil erosion. During focus group discussions and consultations in villages along the roads under study reveal the fact that villagers could identify and could disclose the information on use of only 10 Bryophyte species which are of traditional use (table 2).

Table 1. Distribution of Bryophytes along the roads with their status in IUCN RED List and Catalogue of Life (COL).

\begin{tabular}{|c|c|c|c|c|c|c|}
\hline \multirow[b]{2}{*}{$\begin{array}{l}\text { SI. } \\
\text { No }\end{array}$} & \multirow[b]{2}{*}{$\begin{array}{l}\text { Name of the Bryophyte } \\
\text { species }\end{array}$} & \multirow[b]{2}{*}{ Family } & \multirow[b]{2}{*}{ Habitat and Ecology } & \multirow{2}{*}{$\begin{array}{l}\text { Status as per IUCN } \\
\text { Red list Version } 3.1 \\
\text { an Catalogue of life } \\
\text { annual checklist } 2017\end{array}$} & \multicolumn{2}{|c|}{$\begin{array}{l}\text { Distribution of Bryophytes } \\
\text { along the road }\end{array}$} \\
\hline & & & & & $\begin{array}{l}\text { Aizawl - } \\
\text { Thenzal } \\
\text { Lunglei road }\end{array}$ & $\begin{array}{l}\text { Seling- } \\
\text { Champhai } \\
\text { road }\end{array}$ \\
\hline 1 & $\begin{array}{l}\text { Aneura pinguis (L.) } \\
\text { Dumort. }\end{array}$ & Aneuraceae & $\begin{array}{l}\text { Terrestrial, growing on moist } \\
\text { rocky surface of hills }\end{array}$ & Not listed & + & + \\
\hline 2 & $\begin{array}{l}\text { Riccardia chamedryfolia } \\
\text { (With.) Grolle. }\end{array}$ & Aneuraceae & $\begin{array}{l}\text { Terrestrial, growing on road side } \\
\text { tree bark surface }\end{array}$ & Not listed & + & + \\
\hline 3 & $\begin{array}{l}\text { Anthoceros formosae } \\
\text { Stephani. }\end{array}$ & Anthocerotaceae & $\begin{array}{l}\text { Terrestrial, growing on road side } \\
\text { moist surface of hills }\end{array}$ & Not listed & + & + \\
\hline 4 & $\begin{array}{l}\text { Anthoceros erectus } \\
\text { Kashyap. }\end{array}$ & Anthocerotaceae & $\begin{array}{l}\text { Terrestrial, growing on road side } \\
\text { moist surface of hills }\end{array}$ & Not listed & + & - \\
\hline 5 & $\begin{array}{l}\text { Phaeoceros laevis (L.) } \\
\text { Prosk. }\end{array}$ & Anthocerotaceae & $\begin{array}{l}\text { Terrestrial, growing on moist } \\
\text { surface of road side hills }\end{array}$ & Not listed & + & - \\
\hline 6 & $\begin{array}{l}\text { Asterella khasiana (Griff.) } \\
\text { Grolle. }\end{array}$ & Aytoniaceae & $\begin{array}{l}\text { Terrestrial, growing on road side } \\
\text { moist surface of hills }\end{array}$ & Not listed & - & + \\
\hline 7 & $\begin{array}{l}\text { Plagiochasma } \\
\text { appendiculatum Lehm. \& } \\
\text { Lindenb. }\end{array}$ & Aytoniaceae & $\begin{array}{l}\text { Terrestrial, growing on moist } \\
\text { rocky surface of road side hills }\end{array}$ & Not listed & + & - \\
\hline 8 & $\begin{array}{l}\text { Bartramia subpellucida } \\
\text { Mitt. }\end{array}$ & Bartramiaceae & $\begin{array}{l}\text { Terrestrial, growing on road side } \\
\text { moist surface of hills }\end{array}$ & Not listed & + & - \\
\hline 9 & $\begin{array}{l}\text { Philonotis Fontana } \\
\text { (Hedwig) Bridel. }\end{array}$ & Bartramiaceae & $\begin{array}{l}\text { Terrestrial, growing on moist } \\
\text { surface of road side hills }\end{array}$ & Not listed & - & + \\
\hline 10 & $\begin{array}{l}\text { Eurhynchium riparioides } \\
\text { (Hedw.) P. W. Richards }\end{array}$ & Brachytheciaceae & $\begin{array}{l}\text { Terrestrial, growing on moist } \\
\text { rocky surface of road side hills }\end{array}$ & Not listed & + & - \\
\hline 11 & $\begin{array}{l}\text { Homalothecium sericeum } \\
\text { (Hedw.) Schimp. }\end{array}$ & Brachytheciaceae & $\begin{array}{l}\text { Terrestrial, growing on road side } \\
\text { tree bark surface }\end{array}$ & Not listed & + & + \\
\hline 12 & $\begin{array}{l}\text { Trematodon longicollis } \\
\text { Michx. Hal }\end{array}$ & Bruchiaceae & $\begin{array}{l}\text { Terrestrial, growing on moist } \\
\text { surface of road side hills }\end{array}$ & Not listed & + & - \\
\hline 13 & $\begin{array}{l}\text { Anomobryum filiforme ssp. } \\
\text { concinnatum (Spruc.) } \\
\text { Amann }\end{array}$ & Bryaceae & $\begin{array}{l}\text { Terrestrial, growing on road side } \\
\text { moist surface of hills. }\end{array}$ & Not listed & + & + \\
\hline 14 & $\begin{array}{l}\text { Brachymenium sikkimense } \\
\text { Renauld \& Cardot }\end{array}$ & Bryaceae & $\begin{array}{l}\text { Terrestrial, growing on road side } \\
\text { moist surface of hills }\end{array}$ & Not listed & + & + \\
\hline 15 & $\begin{array}{l}\text { Bryum alpinum Huds. ex. } \\
\text { With. }\end{array}$ & Bryaceae & $\begin{array}{l}\text { Terrestrial, growing on moist } \\
\text { rocky surface of hills }\end{array}$ & Not listed & - & + \\
\hline 16 & $\begin{array}{l}\text { Bryum apiculatum } \\
\text { Schwägr. }\end{array}$ & Bryaceae & $\begin{array}{l}\text { Terrestrial, growing on road side } \\
\text { moist surface of hills }\end{array}$ & Not listed & + & + \\
\hline
\end{tabular}




\begin{tabular}{|c|c|c|c|c|c|c|}
\hline \multirow{2}{*}{$\begin{array}{l}\text { Sl. } \\
\text { No }\end{array}$} & \multirow{2}{*}{$\begin{array}{l}\text { Name of the Bryophyte } \\
\text { species }\end{array}$} & \multirow[b]{2}{*}{ Family } & \multirow[b]{2}{*}{ Habitat and Ecology } & \multirow{2}{*}{$\begin{array}{l}\text { Status as per IUCN } \\
\text { Red list Version } 3.1 \\
\text { an Catalogue of life } \\
\text { annual checklist } 2017\end{array}$} & \multicolumn{2}{|c|}{$\begin{array}{l}\text { Distribution of Bryophytes } \\
\text { along the road }\end{array}$} \\
\hline & & & & & $\begin{array}{l}\text { Aizawl - } \\
\text { Thenzal } \\
\text { Lunglei road }\end{array}$ & $\begin{array}{l}\text { Seling- } \\
\text { Champhai } \\
\text { road }\end{array}$ \\
\hline 17 & Bryum argenteum Hedw. & Bryaceae & $\begin{array}{l}\text { Terrestrial, growing on moist } \\
\text { rocky surface of hills }\end{array}$ & Not listed & 0 & + \\
\hline 18 & Bryum coronatum Schwägr. & Bryaceae & $\begin{array}{l}\text { Terrestrial, growing on moist } \\
\text { surface of hills }\end{array}$ & Not listed & + & + \\
\hline 19 & $\begin{array}{l}\text { Rhodobryum giganteum } \\
\text { (Schwägr.) Paris. }\end{array}$ & Bryaceae & $\begin{array}{l}\text { Terrestrial, growing on moist } \\
\text { surface of road side hills }\end{array}$ & Not listed & + & + \\
\hline 20 & $\begin{array}{l}\text { Calypogeia arguta Nees \& } \\
\text { Mont. ex Nees }\end{array}$ & Calypogeiaceae & $\begin{array}{l}\text { Terrestrial, growing on moist } \\
\text { surface of road side hills }\end{array}$ & Not listed & + & + \\
\hline 21 & $\begin{array}{l}\text { Cephalozia connivens } \\
\text { (Dicks.) Lindb. }\end{array}$ & Cephaloziaceae & $\begin{array}{l}\text { Terrestrial, growing on moist } \\
\text { surface of road side hills }\end{array}$ & Not listed & + & - \\
\hline 22 & $\begin{array}{l}\text { Cyathodium cavernarum } \\
\text { Kunze. }\end{array}$ & Cyathodiaceae & $\begin{array}{l}\text { Terrestrial, growing on moist } \\
\text { rocky surface of road side hills }\end{array}$ & Not listed & + & + \\
\hline 23 & $\begin{array}{l}\text { Cyathophorella tonkinensis } \\
\text { (Broth. \& Paris) Broth. }\end{array}$ & Daltoniaceae & $\begin{array}{l}\text { Terrestrial, growing on road side } \\
\text { tree bark surface }\end{array}$ & Not listed & - & + \\
\hline 24 & Campylopus pilifer Brid. & Dicranaceae & $\begin{array}{l}\text { Terrestrial, growing on moist } \\
\text { surface of road side hills }\end{array}$ & Not listed & + & - \\
\hline 25 & $\begin{array}{l}\text { Campylopus introflexus } \\
\text { (Hedw.) Brid. }\end{array}$ & Dicranaceae & $\begin{array}{l}\text { Terrestrial, growing on moist } \\
\text { surface of road side hills }\end{array}$ & Not listed & + & + \\
\hline 26 & Dicranum flagellare Hedw. & Dicranaceae & $\begin{array}{l}\text { Terrestrial, growing on moist } \\
\text { surface of road side hills }\end{array}$ & Not listed & + & + \\
\hline 27 & $\begin{array}{l}\text { Leucobryum crispum Müll. } \\
\text { Hal }\end{array}$ & . Dicranaceae & $\begin{array}{l}\text { Terrestrial, growing on moist } \\
\text { surface of road side hills }\end{array}$ & Not listed & + & + \\
\hline 28 & $\begin{array}{l}\text { Leucobryum candidum } \\
\text { (Brid. ex P. Beauv.) Wilson }\end{array}$ & Dicranaceae & $\begin{array}{l}\text { Terrestrial, growing on moist } \\
\text { surface of road side hills }\end{array}$ & Not listed & + & + \\
\hline 29 & $\begin{array}{l}\text { Dumortiera hirsuta (Sw.) } \\
\text { Nees). }\end{array}$ & Dumortieraceae & $\begin{array}{l}\text { Terrestrial, growing on moist } \\
\text { rocky surface of road side hills }\end{array}$ & Not listed & + & + \\
\hline 30 & $\begin{array}{l}\text { Erpodium mangiferae } \mathrm{C} . \\
\text { Muell. }\end{array}$ & Erpodiaceae & $\begin{array}{l}\text { Terrestrial, growing on road side } \\
\text { tree bark surface }\end{array}$ & Not listed & + & + \\
\hline 31 & $\begin{array}{l}\text { Fissidens polypodioides } \\
\text { Hedw. }\end{array}$ & Fissidentaceae & $\begin{array}{l}\text { Terrestrial, growing on moist } \\
\text { surface of road side hills }\end{array}$ & Not listed & + & + \\
\hline 32 & Fissidens nobilis Griff. & Fissidentaceae & $\begin{array}{l}\text { Terrestrial, growing on moist } \\
\text { rocky surface of road side hills }\end{array}$ & Not listed & + & + \\
\hline 33 & Frullania acutiloba Mitt. & Frullaniaceae & $\begin{array}{l}\text { Terrestrial, growing on road side } \\
\text { tree bark surface }\end{array}$ & Not listed & + & + \\
\hline 34 & $\begin{array}{l}\text { Frullania tamarisci (L.) } \\
\text { Dumort. }\end{array}$ & Frullaniaceae & $\begin{array}{l}\text { Terrestrial, growing on road side } \\
\text { tree bark surface }\end{array}$ & Not listed & + & + \\
\hline 35 & $\begin{array}{l}\text { Frullania ericoides (Nees) } \\
\text { Mont. }\end{array}$ & Frullaniaceae & $\begin{array}{l}\text { Terrestrial, growing on road side } \\
\text { tree bark surface }\end{array}$ & Not listed & + & + \\
\hline 36 & Frullania neurota Taylor & Frullaniaceae & $\begin{array}{l}\text { Terrestrial, growing on road side } \\
\text { tree bark surface }\end{array}$ & Not listed & + & + \\
\hline 37 & Frullania retusa Mitt. & Frullaniaceae & $\begin{array}{l}\text { Terrestrial, growing on road side } \\
\text { tree bark surface }\end{array}$ & Not listed & + & + \\
\hline 38 & Frullania Raddi & Frullaniaceae & $\begin{array}{l}\text { Terrestrial, growing on moist } \\
\text { surface of road side hills }\end{array}$ & Not listed & + & + \\
\hline 39 & $\begin{array}{l}\text { Frullania mizoramensis } \\
\text { Sushil K. Singh \& } \\
\text { Barbhuiya, sp.nov }\end{array}$ & Frullaniaceae & $\begin{array}{l}\text { Terrestrial, growing on moist } \\
\text { surface of road side hills }\end{array}$ & Not listed & + & + \\
\hline 40 & $\begin{array}{l}\text { Frullania evelynae S. Hatt. } \\
\text { \& Thaith. var. devendrae } \\
\text { Sushil K. Singh \& } \\
\text { Barbhuiya var. nov }\end{array}$ & Frullaniaceae & $\begin{array}{l}\text { Terrestrial, growing on moist } \\
\text { surface of road side hills }\end{array}$ & Not listed & + & + \\
\hline 41 & $\begin{array}{l}\text { Funaria hygrometrica } \\
\text { Hedw. }\end{array}$ & Funariaceae & $\begin{array}{l}\text { Terrestrial, growing on moist } \\
\text { surface of road side hills }\end{array}$ & Not listed & + & + \\
\hline 42 & $\begin{array}{l}\text { Physcomitrium sphaericum } \\
\text { (C. F. Ludw.) Fürnr. }\end{array}$ & Funariaceae & $\begin{array}{l}\text { Terrestrial, growing on moist } \\
\text { surface of road side hills }\end{array}$ & Not listed & + & + \\
\hline 43 & $\begin{array}{l}\text { Physcomitrium pyriforme } \\
\text { (Hedwig) Hampe, Linnaea. }\end{array}$ & Funariaceae & $\begin{array}{l}\text { Terrestrial, growing on moist } \\
\text { surface of road side hills }\end{array}$ & Not listed & + & + \\
\hline 44 & $\begin{array}{l}\text { Jungermania infusca } \\
\text { (Mitt.) Stephani }\end{array}$ & Jungermanniaceae & $\begin{array}{l}\text { Terrestrial, growing on moist } \\
\text { surface of road side hills }\end{array}$ & Not listed & + & + \\
\hline 45 & $\begin{array}{l}\text { Taxiphyllum barbieri } \\
\text { (Cardot \& Copp.) Z. Iwats. }\end{array}$ & Hypnaceae & $\begin{array}{l}\text { Terrestrial, growing on moist } \\
\text { rocky surface of road side hills }\end{array}$ & Not listed & + & + \\
\hline 46 & $\begin{array}{l}\text { Lejeunea flava (Sw.) Nees } \\
\text { Lejeuneaceae }\end{array}$ & Lejeuneaceae & $\begin{array}{l}\text { Terrestrial, growing on road side } \\
\text { tree bark surface }\end{array}$ & Not listed & + & + \\
\hline
\end{tabular}




\begin{tabular}{|c|c|c|c|c|c|c|}
\hline \multirow[b]{2}{*}{$\begin{array}{l}\text { SI. } \\
\text { No }\end{array}$} & \multirow[b]{2}{*}{$\begin{array}{l}\text { Name of the Bryophyte } \\
\text { species }\end{array}$} & \multirow[b]{2}{*}{ Family } & \multirow[b]{2}{*}{ Habitat and Ecology } & \multirow{2}{*}{$\begin{array}{l}\text { Status as per IUCN } \\
\text { Red list Version } 3.1 \\
\text { an Catalogue of life } \\
\text { annual checklist } 2017\end{array}$} & \multicolumn{2}{|c|}{$\begin{array}{l}\text { Distribution of Bryophytes } \\
\text { along the road }\end{array}$} \\
\hline & & & & & $\begin{array}{l}\text { Aizawl - } \\
\text { Thenzal } \\
\text { Lunglei road } \\
\end{array}$ & $\begin{array}{l}\text { Seling- } \\
\text { Champhai } \\
\text { road }\end{array}$ \\
\hline 47 & $\begin{array}{l}\text { Ptychanthus striatus } \\
\text { (Lehm. et Lindenb.) Nee. }\end{array}$ & Lejeuneaceae & $\begin{array}{l}\text { Terrestrial, growing on road side } \\
\text { tree bark surface }\end{array}$ & Not listed & 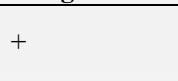 & + \\
\hline 48 & $\begin{array}{l}\text { Marchantia paleacea } \\
\text { Bertol. }\end{array}$ & Marchantiaceae & $\begin{array}{l}\text { Terrestrial, growing on moist } \\
\text { surface of road side hills }\end{array}$ & Not listed & + & + \\
\hline 49 & $\begin{array}{l}\text { Marchantia subintegra } \\
\text { Mitt. }\end{array}$ & Marchantiaceae & $\begin{array}{l}\text { Terrestrial, growing on moist } \\
\text { surface of road side hills }\end{array}$ & Not listed & + & + \\
\hline 50 & $\begin{array}{l}\text { Marchantia emarginata } \\
\text { Reinw., Blume \& Nees. }\end{array}$ & Marchantiaceae & $\begin{array}{l}\text { Terrestrial, growing on moist } \\
\text { surface of road side hills }\end{array}$ & Not listed & + & + \\
\hline 51 & $\begin{array}{l}\text { Dumortiera hirsuta (Sw.) } \\
\text { Nees }\end{array}$ & Marchantiaceae & $\begin{array}{l}\text { Terrestrial, growing on moist } \\
\text { rocky surface of hills }\end{array}$ & Not listed & + & + \\
\hline 52 & $\begin{array}{l}\text { Papillaria leuconeura } \\
\text { (Müll. Hal.) A. Jaeger }\end{array}$ & Meteoriaceae & $\begin{array}{l}\text { Terrestrial, growing on road side } \\
\text { tree bark surface }\end{array}$ & Not listed & + & + \\
\hline 53 & $\begin{array}{l}\text { Meteoriopsis reclinata } \\
\text { (Müll. Hal.) M. Fleisch. }\end{array}$ & Meteorioceae & $\begin{array}{l}\text { Terrestrial, growing on road side } \\
\text { tree bark surface }\end{array}$ & Not listed & + & + \\
\hline 54 & $\begin{array}{l}\text { Meteoriopsis squarrosa } \\
\text { (Hook. ex Harv.) M. } \\
\text { Fleisch. }\end{array}$ & Meteorioceae & $\begin{array}{l}\text { Terrestrial, growing on road side } \\
\text { tree bark surface }\end{array}$ & Not listed & + & + \\
\hline 55 & $\begin{array}{l}\text { Metzgeria conjugata } \\
\text { Lindb. }\end{array}$ & Metzgeriaceeae & $\begin{array}{l}\text { Terrestrial, growing on moist } \\
\text { rocky surface of road side hills }\end{array}$ & Not listed & + & + \\
\hline 56 & $\begin{array}{l}\text { Plagiomnium ellipticum } \\
\text { (Brid.) T. Kop. }\end{array}$ & Mniaceae & $\begin{array}{l}\text { Terrestrial, growing on moist } \\
\text { surface of road side hills }\end{array}$ & Not listed & + & + \\
\hline 57 & $\begin{array}{l}\text { Neckeropsis undulata } \\
\text { (Hedw.) Reichardt }\end{array}$ & Neckeraceae & $\begin{array}{l}\text { Terrestrial, growing on moist } \\
\text { surface of road side hills }\end{array}$ & Not listed & + & + \\
\hline 58 & $\begin{array}{l}\text { Octoblepharum albidum } \\
\text { Hedw. }\end{array}$ & Octoblepharaceae & $\begin{array}{l}\text { Terrestrial, growing on road side } \\
\text { tree bark surface }\end{array}$ & Not listed & + & + \\
\hline 59 & $\begin{array}{l}\text { Pallavicinia lyellii (Hook.) } \\
\text { Gray. }\end{array}$ & Pallaviciniaceae & $\begin{array}{l}\text { Terrestrial, growing on moist } \\
\text { surface of road side hills }\end{array}$ & Not listed & + & + \\
\hline 60 & Pellia epiphylla (L.) Corda & Pelliaceae & $\begin{array}{l}\text { Terrestrial, growing on moist } \\
\text { rocky surface of road side hills }\end{array}$ & Not listed & + & + \\
\hline 61 & $\begin{array}{l}\text { Plagiochila spinulosa } \\
\text { (Dicks.) Dumort. }\end{array}$ & Plagiochilaceae & $\begin{array}{l}\text { Terrestrial, growing on road side } \\
\text { tree bark surface }\end{array}$ & Not listed & + & + \\
\hline 62 & $\begin{array}{l}\text { Atrichum undulatum } \\
\text { (Hedw.) P. Beauv. }\end{array}$ & Polytrichaceae & $\begin{array}{l}\text { Terrestrial, growing on moist } \\
\text { surface of road side hills }\end{array}$ & Not listed & + & + \\
\hline 63 & $\begin{array}{l}\text { Hyophila involuta (Hook.) } \\
\text { A. Jaeger }\end{array}$ & Pottiaceae & $\begin{array}{l}\text { Terrestrial, growing on moist } \\
\text { rocky surface of road side hills }\end{array}$ & Not listed & + & + \\
\hline 64 & $\begin{array}{l}\text { Pogonatum aloides (Hedw.) } \\
\text { P. Beauv. }\end{array}$ & Polytrichaceae & $\begin{array}{l}\text { Terrestrial, growing on moist } \\
\text { surface of road side hills }\end{array}$ & Not listed & + & + \\
\hline 65 & $\begin{array}{l}\text { Pogonatum contortum } \\
\text { (Menzies ex Brid.) Lesq. }\end{array}$ & Polytrichaceae & $\begin{array}{l}\text { Terrestrial, growing on moist } \\
\text { surface of road side hills }\end{array}$ & Not listed & + & + \\
\hline 66 & $\begin{array}{l}\text { Porella campylophylla } \\
\text { (Lehm. \& Lindenb.) Trevis. }\end{array}$ & Porellaceae & $\begin{array}{l}\text { Terrestrial, growing on road side } \\
\text { tree bark surface }\end{array}$ & Not listed & + & + \\
\hline 67 & Barbula convoluta Hedw. & Pottiaceae & $\begin{array}{l}\text { Terrestrial, growing on moist } \\
\text { rocky surface of hills }\end{array}$ & Not listed & + & + \\
\hline 68 & $\begin{array}{l}\text { Racopilum cuspidigerum } \\
\text { (Schwägr.) Ångström. }\end{array}$ & Racopilaceae & $\begin{array}{l}\text { Terrestrial, growing on road side } \\
\text { tree bark surface }\end{array}$ & Not listed & + & + \\
\hline 69 & $\begin{array}{l}\text { Radula complanata }(\mathrm{L} .) \\
\text { Dumort. Tree bark }\end{array}$ & Radulaceae & $\begin{array}{l}\text { Terrestrial, growing on road side } \\
\text { tree bark surface }\end{array}$ & Not listed & + & + \\
\hline 70 & $\begin{array}{l}\text { Riccia discolour Lehm. \& } \\
\text { Lindenb. }\end{array}$ & Ricciaceae & $\begin{array}{l}\text { Terrestrial, growing on moist } \\
\text { surface of road side hills }\end{array}$ & Not listed & + & + \\
\hline 71 & Riccia glauca L. Soil & Ricciaceae & $\begin{array}{l}\text { Terrestrial, growing on moist } \\
\text { surface of road side hills }\end{array}$ & Not listed & + & + \\
\hline 72 & $\begin{array}{l}\text { Riccia fluitans } \mathrm{L} \text {. } \\
\text { Ricciaceae }\end{array}$ & Ricciaceae & $\begin{array}{l}\text { Terrestrial, growing on road side } \\
\text { water logged area (submerged ) }\end{array}$ & Not listed & + & + \\
\hline 73 & $\begin{array}{l}\text { Stereophyllum anceps } \\
\text { (Bosch \& Sande Lac.) } \\
\text { Broth. }\end{array}$ & Stereophyllaceae & $\begin{array}{l}\text { Terrestrial, growing on moist } \\
\text { surface of road side hills }\end{array}$ & Not listed & + & + \\
\hline 74 & Targionia hypophylla $\mathrm{L}$. & Targionioceae & $\begin{array}{l}\text { Terrestrial, growing on moist } \\
\text { rocky surface of road side hills }\end{array}$ & Not listed & + & - \\
\hline 75 & $\begin{array}{l}\text { Thuidium cymbifolium } \\
\text { (Dozy \& Molk.) Dozy \& } \\
\text { Molk. }\end{array}$ & Thuidiaceae & $\begin{array}{l}\text { Terrestrial, growing on moist } \\
\text { surface of road side hills }\end{array}$ & Not listed & + & - \\
\hline 76 & $\begin{array}{l}\text { Thuidium delicatulum } \\
\text { (Hedw.) Schimp. }\end{array}$ & Thuidiaceae & $\begin{array}{l}\text { Terrestrial, growing on moist } \\
\text { surface of road side hills }\end{array}$ & Not listed & + & + \\
\hline
\end{tabular}


Table 1. Continued.

\begin{tabular}{|c|c|c|c|c|c|c|}
\hline \multirow[b]{2}{*}{$\begin{array}{l}\text { SI. } \\
\text { No }\end{array}$} & \multirow[b]{2}{*}{$\begin{array}{l}\text { Name of the Bryophyte } \\
\text { species }\end{array}$} & \multirow[b]{2}{*}{ Family } & \multirow[b]{2}{*}{ Habitat and Ecology } & \multirow{2}{*}{$\begin{array}{l}\text { Status as per IUCN } \\
\text { Red list Version } 3.1 \\
\text { an Catalogue of life } \\
\text { annual checklist } \\
2017\end{array}$} & \multicolumn{2}{|c|}{$\begin{array}{l}\text { Distribution of Bryophytes } \\
\text { along the road }\end{array}$} \\
\hline & & & & & $\begin{array}{l}\text { Thenzal- } \\
\text { Serchip } \\
\text { road }\end{array}$ & $\begin{array}{l}\text { Lunglei-Tlabung- } \\
\text { Kwarpuicchua } \\
\text { road }\end{array}$ \\
\hline 1 & Aneura pinguis (L.) Dumort. & Aneuraceae & $\begin{array}{l}\text { Terrestrial, growing on moist } \\
\text { rocky surface of hills }\end{array}$ & Not listed & - & + \\
\hline 2 & $\begin{array}{l}\text { Riccardia chamedryfolia } \\
\text { (With.) Grolle. }\end{array}$ & Aneuraceae & $\begin{array}{l}\text { Terrestrial, growing on road side } \\
\text { tree bark surface }\end{array}$ & Not listed & + & + \\
\hline 3 & $\begin{array}{l}\text { Anthoceros formosae } \\
\text { Stephani. }\end{array}$ & Anthocerotaceae & $\begin{array}{l}\text { Terrestrial, growing on road side } \\
\text { moist surface of hills }\end{array}$ & Not listed & + & + \\
\hline 4 & Anthoceros erectus Kashyap. & Anthocerotaceae & $\begin{array}{l}\text { Terrestrial, growing on road side } \\
\text { moist surface of hills }\end{array}$ & Not listed & + & + \\
\hline 5 & Phaeoceros laevis (L.) Prosk. & Anthocerotaceae & $\begin{array}{l}\text { Terrestrial, growing on moist } \\
\text { surface of road side hills }\end{array}$ & Not listed & + & + \\
\hline 6 & $\begin{array}{l}\text { Asterella khasiana (Griff.) } \\
\text { Grolle. }\end{array}$ & Aytoniaceae & $\begin{array}{l}\text { Terrestrial, growing on road side } \\
\text { moist surface of hills }\end{array}$ & Not listed & + & + \\
\hline 7 & $\begin{array}{l}\text { Plagiochasma } \\
\text { appendiculatum Lehm. \& } \\
\text { Lindenb. }\end{array}$ & Aytoniaceae & $\begin{array}{l}\text { Terrestrial, growing on moist } \\
\text { rocky surface of road side hills }\end{array}$ & Not listed & + & + \\
\hline 8 & Bartramia subpellucida Mitt. & Bartramiaceae & $\begin{array}{l}\text { Terrestrial, growing on road side } \\
\text { moist surface of hills }\end{array}$ & Not listed & + & + \\
\hline 9 & $\begin{array}{l}\text { Philonotis Fontana (Hedwig) } \\
\text { Bridel. }\end{array}$ & Bartramiaceae & $\begin{array}{l}\text { Terrestrial, growing on moist } \\
\text { surface of road side hills }\end{array}$ & Not listed & + & - \\
\hline 10 & $\begin{array}{l}\text { Eurhynchium riparioides } \\
\text { (Hedw.) P. W. Richards }\end{array}$ & $\begin{array}{l}\text { Brachytheciacea } \\
\text { e }\end{array}$ & $\begin{array}{l}\text { Terrestrial, growing on moist } \\
\text { rocky surface of road side hills }\end{array}$ & Not listed & + & + \\
\hline 11 & $\begin{array}{l}\text { Homalothecium sericeum } \\
\text { (Hedw.) Schimp. }\end{array}$ & $\begin{array}{l}\text { Brachytheciacea } \\
\text { e }\end{array}$ & $\begin{array}{l}\text { Terrestrial, growing on road side } \\
\text { tree bark surface }\end{array}$ & Not listed & + & + \\
\hline 12 & $\begin{array}{l}\text { Trematodon longicollis } \\
\text { Michx. Hal }\end{array}$ & Bruchiaceae & $\begin{array}{l}\text { Terrestrial, growing on moist } \\
\text { surface of road side hills }\end{array}$ & Not listed & + & + \\
\hline 13 & $\begin{array}{l}\text { Anomobryum filiforme ssp. } \\
\text { concinnatum (Spruc.) Amann }\end{array}$ & Bryaceae & $\begin{array}{l}\text { Terrestrial, growing on road side } \\
\text { moist surface of hills. }\end{array}$ & Not listed & + & + \\
\hline 14 & $\begin{array}{l}\text { Brachymenium sikkimense } \\
\text { Renauld \& Cardot }\end{array}$ & Bryaceae & $\begin{array}{l}\text { Terrestrial, growing on road side } \\
\text { moist surface of hills }\end{array}$ & Not listed & - & + \\
\hline 15 & $\begin{array}{l}\text { Bryum alpinum Huds. ex. } \\
\text { With. }\end{array}$ & Bryaceae & $\begin{array}{l}\text { Terrestrial, growing on moist } \\
\text { rocky surface of hills }\end{array}$ & Not listed & + & + \\
\hline 16 & Bryum apiculatum Schwägr. & Bryaceae & $\begin{array}{l}\text { Terrestrial, growing on road side } \\
\text { moist surface of hills }\end{array}$ & Not listed & - & + \\
\hline 17 & Bryum argenteum Hedw. & Bryaceae & $\begin{array}{l}\text { Terrestrial, growing on moist } \\
\text { rocky surface of hills }\end{array}$ & Not listed & + & + \\
\hline 18 & Bryum coronatum Schwägr. & Bryaceae & $\begin{array}{l}\text { Terrestrial, growing on moist } \\
\text { surface of hills }\end{array}$ & Not listed & + & + \\
\hline 19 & $\begin{array}{l}\text { Rhodobryum giganteum } \\
\text { (Schwägr.) Paris. }\end{array}$ & Bryaceae & $\begin{array}{l}\text { Terrestrial, growing on moist } \\
\text { surface of road side hills }\end{array}$ & Not listed & + & - \\
\hline 20 & $\begin{array}{l}\text { Calypogeia arguta Nees \& } \\
\text { Mont. ex Nees }\end{array}$ & Calypogeiaceae & $\begin{array}{l}\text { Terrestrial, growing on moist } \\
\text { surface of road side hills }\end{array}$ & Not listed & + & + \\
\hline 21 & $\begin{array}{l}\text { Cephalozia connivens } \\
\text { (Dicks.) Lindb. }\end{array}$ & Cephaloziaceae & $\begin{array}{l}\text { Terrestrial, growing on moist } \\
\text { surface of road side hills }\end{array}$ & Not listed & + & + \\
\hline 22 & $\begin{array}{l}\text { Cyathodium cavernarum } \\
\text { Kunze. }\end{array}$ & Cyathodiaceae & $\begin{array}{l}\text { Terrestrial, growing on moist } \\
\text { rocky surface of road side hills }\end{array}$ & Not listed & + & + \\
\hline 23 & $\begin{array}{l}\text { Cyathophorella tonkinensis } \\
\text { (Broth. \& Paris) Broth. }\end{array}$ & Daltoniaceae & $\begin{array}{l}\text { Terrestrial, growing on road side } \\
\text { tree bark surface }\end{array}$ & Not listed & + & + \\
\hline 24 & Campylopus pilifer Brid. & Dicranaceae & $\begin{array}{l}\text { Terrestrial, growing on moist } \\
\text { surface of road side hills }\end{array}$ & Not listed & + & + \\
\hline 25 & $\begin{array}{l}\text { Campylopus introflexus } \\
\text { (Hedw.) Brid. }\end{array}$ & Dicranaceae & $\begin{array}{l}\text { Terrestrial, growing on moist } \\
\text { surface of road side hills }\end{array}$ & Not listed & - & + \\
\hline 26 & Dicranum flagellare Hedw. & Dicranaceae & $\begin{array}{l}\text { Terrestrial, growing on moist } \\
\text { surface of road side hills }\end{array}$ & Not listed & + & + \\
\hline 27 & $\begin{array}{l}\text { Leucobryum crispum Müll. } \\
\text { Hal }\end{array}$ & Dicranaceae & $\begin{array}{l}\text { Terrestrial, growing on moist } \\
\text { surface of road side hills }\end{array}$ & Not listed & + & + \\
\hline 28 & $\begin{array}{l}\text { Leucobryum candidum (Brid. } \\
\text { ex P. Beauv.) Wilson }\end{array}$ & Dicranaceae & $\begin{array}{l}\text { Terrestrial, growing on moist } \\
\text { surface of road side hills }\end{array}$ & Not listed & + & + \\
\hline 29 & $\begin{array}{l}\text { Dumortiera hirsuta (Sw.) } \\
\text { Nees). }\end{array}$ & Dumortieraceae & $\begin{array}{l}\text { Terrestrial, growing on moist } \\
\text { rocky surface of road side hills }\end{array}$ & Not listed & + & + \\
\hline 30 & $\begin{array}{l}\text { Erpodium mangiferae } \mathrm{C} . \\
\text { Muell. }\end{array}$ & Erpodiaceae & $\begin{array}{l}\text { Terrestrial, growing on road side } \\
\text { tree bark surface }\end{array}$ & Not listed & + & - \\
\hline 31 & Fissidens polypodioides & Fissidentaceae & Terrestrial, growing on moist & Not listed & + & + \\
\hline
\end{tabular}




\begin{tabular}{|c|c|c|c|c|c|c|}
\hline \multirow[b]{2}{*}{$\begin{array}{l}\text { Sl. } \\
\text { No }\end{array}$} & \multirow[b]{2}{*}{$\begin{array}{l}\text { Name of the Bryophyte } \\
\text { species }\end{array}$} & \multirow[b]{2}{*}{ Family } & \multirow[b]{2}{*}{ Habitat and Ecology } & \multirow{2}{*}{$\begin{array}{l}\text { Status as per IUCN } \\
\text { Red list Version } 3.1 \\
\text { an Catalogue of life } \\
\text { annual checklist } \\
2017\end{array}$} & \multicolumn{2}{|c|}{$\begin{array}{l}\text { Distribution of Bryophytes } \\
\text { along the road }\end{array}$} \\
\hline & & & & & $\begin{array}{l}\text { Thenzal- } \\
\text { Serchip } \\
\text { road }\end{array}$ & $\begin{array}{l}\text { Lunglei-Tlabung- } \\
\text { Kwarpuicchua } \\
\text { road }\end{array}$ \\
\hline & Hedw. & & surface of road side hills & & & \\
\hline 32 & Fissidens nobilis Griff. & Fissidentaceae & $\begin{array}{l}\text { Terrestrial, growing on moist } \\
\text { rocky surface of road side hills }\end{array}$ & Not listed & + & - \\
\hline 33 & Frullania acutiloba Mitt. & Frullaniaceae & $\begin{array}{l}\text { Terrestrial, growing on road side } \\
\text { tree bark surface }\end{array}$ & Not listed & + & + \\
\hline 34 & $\begin{array}{l}\text { Frullania tamarisci (L.) } \\
\text { Dumort. }\end{array}$ & Frullaniaceae & $\begin{array}{l}\text { Terrestrial, growing on road side } \\
\text { tree bark surface }\end{array}$ & Not listed & + & - \\
\hline 35 & $\begin{array}{l}\text { Frullania ericoides (Nees) } \\
\text { Mont. }\end{array}$ & Frullaniaceae & $\begin{array}{l}\text { Terrestrial, growing on road side } \\
\text { tree bark surface }\end{array}$ & Not listed & + & - \\
\hline 36 & Frullania neurota Taylor & Frullaniaceae & $\begin{array}{l}\text { Terrestrial, growing on road side } \\
\text { tree bark surface }\end{array}$ & Not listed & + & - \\
\hline 37 & Frullania retusa Mitt. & Frullaniaceae & $\begin{array}{l}\text { Terrestrial, growing on road side } \\
\text { tree bark surface }\end{array}$ & Not listed & + & + \\
\hline 38 & Frullania Raddi & Frullaniaceae & $\begin{array}{l}\text { Terrestrial, growing on moist } \\
\text { surface of road side hills }\end{array}$ & Not listed & + & - \\
\hline 39 & $\begin{array}{l}\text { Frullania mizoramensis } \\
\text { Sushil K. Singh \& Barbhuiya, } \\
\text { sp. nov }\end{array}$ & Frullaniaceae & $\begin{array}{l}\text { Terrestrial, growing on moist } \\
\text { surface of road side hills }\end{array}$ & Not listed & + & + \\
\hline 40 & $\begin{array}{l}\text { Frullania evelynae S. Hatt. \& } \\
\text { Thaith. var. devendrae Sushil } \\
\text { K. Singh \& Barbhuiya var. } \\
\text { nov }\end{array}$ & Frullaniaceae & $\begin{array}{l}\text { Terrestrial, growing on moist } \\
\text { surface of road side hills }\end{array}$ & Not listed & + & - \\
\hline 41 & Funaria hygrometrica Hedw. & Funariaceae & $\begin{array}{l}\text { Terrestrial, growing on moist } \\
\text { surface of road side hills }\end{array}$ & Not listed & + & + \\
\hline 42 & $\begin{array}{l}\text { Physcomitrium sphaericum } \\
\text { (C. F. Ludw.) Fürnr. }\end{array}$ & Funariaceae & $\begin{array}{l}\text { Terrestrial, growing on moist } \\
\text { surface of road side hills }\end{array}$ & Not listed & + & - \\
\hline 43 & $\begin{array}{l}\text { Physcomitrium pyriforme } \\
\text { (Hedwig) Hampe, Linnaea. }\end{array}$ & Funariaceae & $\begin{array}{l}\text { Terrestrial, growing on moist } \\
\text { surface of road side hills }\end{array}$ & Not listed & + & - \\
\hline 44 & $\begin{array}{l}\text { Jungermania infusca (Mitt.) } \\
\text { Stephani }\end{array}$ & $\begin{array}{l}\text { Jungermanniace } \\
\text { ae }\end{array}$ & $\begin{array}{l}\text { Terrestrial, growing on moist } \\
\text { surface of road side hills }\end{array}$ & Not listed & + & + \\
\hline 45 & $\begin{array}{l}\text { Taxiphyllum barbieri (Cardot } \\
\& \text { Copp.) Z. Iwats. }\end{array}$ & Hypnaceae & $\begin{array}{l}\text { Terrestrial, growing on moist } \\
\text { rocky surface of road side hills }\end{array}$ & Not listed & + & + \\
\hline 46 & $\begin{array}{l}\text { Lejeunea flava (Sw.) Nees } \\
\text { Lejeuneaceae }\end{array}$ & Lejeuneaceae & $\begin{array}{l}\text { Terrestrial, growing on road side } \\
\text { tree bark surface }\end{array}$ & Not listed & + & + \\
\hline 47 & $\begin{array}{l}\text { Ptychanthus striatus (Lehm. } \\
\text { et Lindenb.) Nee. }\end{array}$ & Lejeuneaceae & $\begin{array}{l}\text { Terrestrial, growing on road side } \\
\text { tree bark surface }\end{array}$ & Not listed & + & - \\
\hline 48 & Marchantia paleacea Bertol. & Marchantiaceae & $\begin{array}{l}\text { Terrestrial, growing on moist } \\
\text { surface of road side hills }\end{array}$ & Not listed & + & + \\
\hline 49 & Marchantia subintegra Mitt. & Marchantiaceae & $\begin{array}{l}\text { Terrestrial, growing on moist } \\
\text { surface of road side hills }\end{array}$ & Not listed & + & + \\
\hline 50 & $\begin{array}{l}\text { Marchantia emarginata } \\
\text { Reinw., Blume \& Nees. }\end{array}$ & Marchantiaceae & $\begin{array}{l}\text { Terrestrial, growing on moist } \\
\text { surface of road side hills }\end{array}$ & Not listed & + & + \\
\hline 51 & $\begin{array}{l}\text { Dumortiera hirsuta (Sw.) } \\
\text { Nees }\end{array}$ & Marchantiaceae & $\begin{array}{l}\text { Terrestrial, growing on moist } \\
\text { rocky surface of hills }\end{array}$ & Not listed & + & + \\
\hline 52 & $\begin{array}{l}\text { Papillaria leuconeura (Müll. } \\
\text { Hal.) A. Jaeger }\end{array}$ & Meteoriaceae & $\begin{array}{l}\text { Terrestrial, growing on road side } \\
\text { tree bark surface }\end{array}$ & Not listed & + & + \\
\hline 53 & $\begin{array}{l}\text { Meteoriopsis reclinata (Müll. } \\
\text { Hal.) M. Fleisch. }\end{array}$ & Meteorioceae & $\begin{array}{l}\text { Terrestrial, growing on road side } \\
\text { tree bark surface }\end{array}$ & Not listed & + & - \\
\hline 54 & $\begin{array}{l}\text { Meteoriopsis squarrosa } \\
\text { (Hook. ex Harv.) M. Fleisch. }\end{array}$ & Meteorioceae & $\begin{array}{l}\text { Terrestrial, growing on road side } \\
\text { tree bark surface }\end{array}$ & Not listed & + & + \\
\hline 55 & Metzgeria conjugata Lindb. & Metzgeriaceeae & $\begin{array}{l}\text { Terrestrial, growing on moist } \\
\text { rocky surface of road side hills }\end{array}$ & Not listed & + & - \\
\hline 56 & $\begin{array}{l}\text { Plagiomnium ellipticum } \\
\text { (Brid.) T. Kop. }\end{array}$ & Mniaceae & $\begin{array}{l}\text { Terrestrial, growing on moist } \\
\text { surface of road side hills }\end{array}$ & Not listed & + & + \\
\hline 57 & $\begin{array}{l}\text { Neckeropsis undulata (Hedw.) } \\
\text { Reichardt }\end{array}$ & Neckeraceae & $\begin{array}{l}\text { Terrestrial, growing on moist } \\
\text { surface of road side hills }\end{array}$ & Not listed & + & + \\
\hline 58 & $\begin{array}{l}\text { Octoblepharum albidum } \\
\text { Hedw. }\end{array}$ & $\begin{array}{l}\text { Octoblepharacea } \\
\text { e }\end{array}$ & $\begin{array}{l}\text { Terrestrial, growing on road side } \\
\text { tree bark surface }\end{array}$ & Not listed & + & + \\
\hline 59 & $\begin{array}{l}\text { Pallavicinia lyellii (Hook.) } \\
\text { Gray. }\end{array}$ & Pallaviciniaceae & $\begin{array}{l}\text { Terrestrial, growing on moist } \\
\text { surface of road side hills }\end{array}$ & Not listed & + & - \\
\hline 60 & Pellia epiphylla (L.) Corda & Pelliaceae & $\begin{array}{l}\text { Terrestrial, growing on moist } \\
\text { rocky surface of road side hills }\end{array}$ & Not listed & + & + \\
\hline
\end{tabular}




\begin{tabular}{|c|c|c|c|c|c|c|}
\hline \multirow[b]{2}{*}{$\begin{array}{l}\text { Sl. } \\
\text { No }\end{array}$} & \multirow[b]{2}{*}{$\begin{array}{l}\text { Name of the Bryophyte } \\
\text { species }\end{array}$} & \multirow[b]{2}{*}{ Family } & \multirow[b]{2}{*}{ Habitat and Ecology } & \multirow{2}{*}{$\begin{array}{l}\text { Status as per IUCN } \\
\text { Red list Version } 3.1 \\
\text { an Catalogue of life } \\
\text { annual checklist } \\
2017\end{array}$} & \multicolumn{2}{|c|}{$\begin{array}{l}\text { Distribution of Bryophytes } \\
\text { along the road }\end{array}$} \\
\hline & & & & & $\begin{array}{l}\text { Thenzal- } \\
\text { Serchip } \\
\text { road }\end{array}$ & $\begin{array}{l}\text { Lunglei-Tlabung- } \\
\text { Kwarpuicchua } \\
\text { road }\end{array}$ \\
\hline 61 & $\begin{array}{l}\text { Plagiochila spinulosa } \\
\text { (Dicks.) Dumort. }\end{array}$ & Plagiochilaceae & $\begin{array}{l}\text { Terrestrial, growing on road side } \\
\text { tree bark surface }\end{array}$ & Not listed & + & 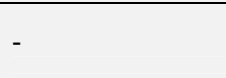 \\
\hline 62 & $\begin{array}{l}\text { Atrichum undulatum (Hedw.) } \\
\text { P. Beauv. }\end{array}$ & Polytrichaceae & $\begin{array}{l}\text { Terrestrial, growing on moist } \\
\text { surface of road side hills }\end{array}$ & Not listed & - & + \\
\hline 63 & $\begin{array}{l}\text { Hyophila involuta (Hook.) A. } \\
\text { Jaeger }\end{array}$ & Pottiaceae & $\begin{array}{l}\text { Terrestrial, growing on moist } \\
\text { rocky surface of road side hills }\end{array}$ & Not listed & + & - \\
\hline 64 & $\begin{array}{l}\text { Pogonatum aloides (Hedw.) } \\
\text { P. Beauv. }\end{array}$ & Polytrichaceae & $\begin{array}{l}\text { Terrestrial, growing on moist } \\
\text { surface of road side hills }\end{array}$ & Not listed & + & - \\
\hline 65 & $\begin{array}{l}\text { Pogonatum contortum } \\
\text { (Menzies ex Brid.) Lesq. }\end{array}$ & Polytrichaceae & $\begin{array}{l}\text { Terrestrial, growing on moist } \\
\text { surface of road side hills }\end{array}$ & Not listed & + & - \\
\hline 66 & $\begin{array}{l}\text { Porella campylophylla } \\
\text { (Lehm. \& Lindenb.) Trevis. }\end{array}$ & Porellaceae & $\begin{array}{l}\text { Terrestrial, growing on road side } \\
\text { tree bark surface }\end{array}$ & Not listed & + & + \\
\hline 67 & Barbula convoluta Hedw. & Pottiaceae & $\begin{array}{l}\text { Terrestrial, growing on moist } \\
\text { rocky surface of hills }\end{array}$ & Not listed & - & + \\
\hline 68 & $\begin{array}{l}\text { Racopilum cuspidigerum } \\
\text { (Schwägr.) Ångström. }\end{array}$ & Racopilaceae & $\begin{array}{l}\text { Terrestrial, growing on road side } \\
\text { tree bark surface }\end{array}$ & Not listed & + & + \\
\hline 69 & $\begin{array}{l}\text { Radula complanata (L.) } \\
\text { Dumort. Tree bark }\end{array}$ & Radulaceae & $\begin{array}{l}\text { Terrestrial, growing on road side } \\
\text { tree bark surface }\end{array}$ & Not listed & + & - \\
\hline 70 & $\begin{array}{l}\text { Riccia discolour Lehm. \& } \\
\text { Lindenb. }\end{array}$ & Ricciaceae & $\begin{array}{l}\text { Terrestrial, growing on moist } \\
\text { surface of road side hills }\end{array}$ & Not listed & + & + \\
\hline 71 & Riccia glauca L. Soil & Ricciaceae & $\begin{array}{l}\text { Terrestrial, growing on moist } \\
\text { surface of road side hills }\end{array}$ & Not listed & + & + \\
\hline 72 & Riccia fluitans $\mathrm{L}$. Ricciaceae & Ricciaceae & $\begin{array}{l}\text { Terrestrial, growing on road side } \\
\text { water logged area (submerged ) }\end{array}$ & Not listed & + & - \\
\hline 73 & $\begin{array}{l}\text { Stereophyllum anceps (Bosch } \\
\& \text { Sande Lac.) Broth. }\end{array}$ & Stereophyllaceae & $\begin{array}{l}\text { Terrestrial, growing on moist } \\
\text { surface of road side hills }\end{array}$ & Not listed & - & + \\
\hline 74 & Targionia hypophylla L. & Targionioceae & $\begin{array}{l}\text { Terrestrial, growing on moist } \\
\text { rocky surface of road side hills }\end{array}$ & Not listed & + & + \\
\hline 75 & $\begin{array}{l}\text { Thuidium cymbifolium (Dozy } \\
\& \text { Molk.) Dozy \& Molk. }\end{array}$ & Thuidiaceae & $\begin{array}{l}\text { Terrestrial, growing on moist } \\
\text { surface of road side hills }\end{array}$ & Not listed & - & + \\
\hline 76 & $\begin{array}{l}\text { Thuidium delicatulum } \\
\text { (Hedw.) Schimp. }\end{array}$ & Thuidiaceae & $\begin{array}{l}\text { Terrestrial, growing on moist } \\
\text { surface of road side hills }\end{array}$ & Not listed & + & + \\
\hline
\end{tabular}

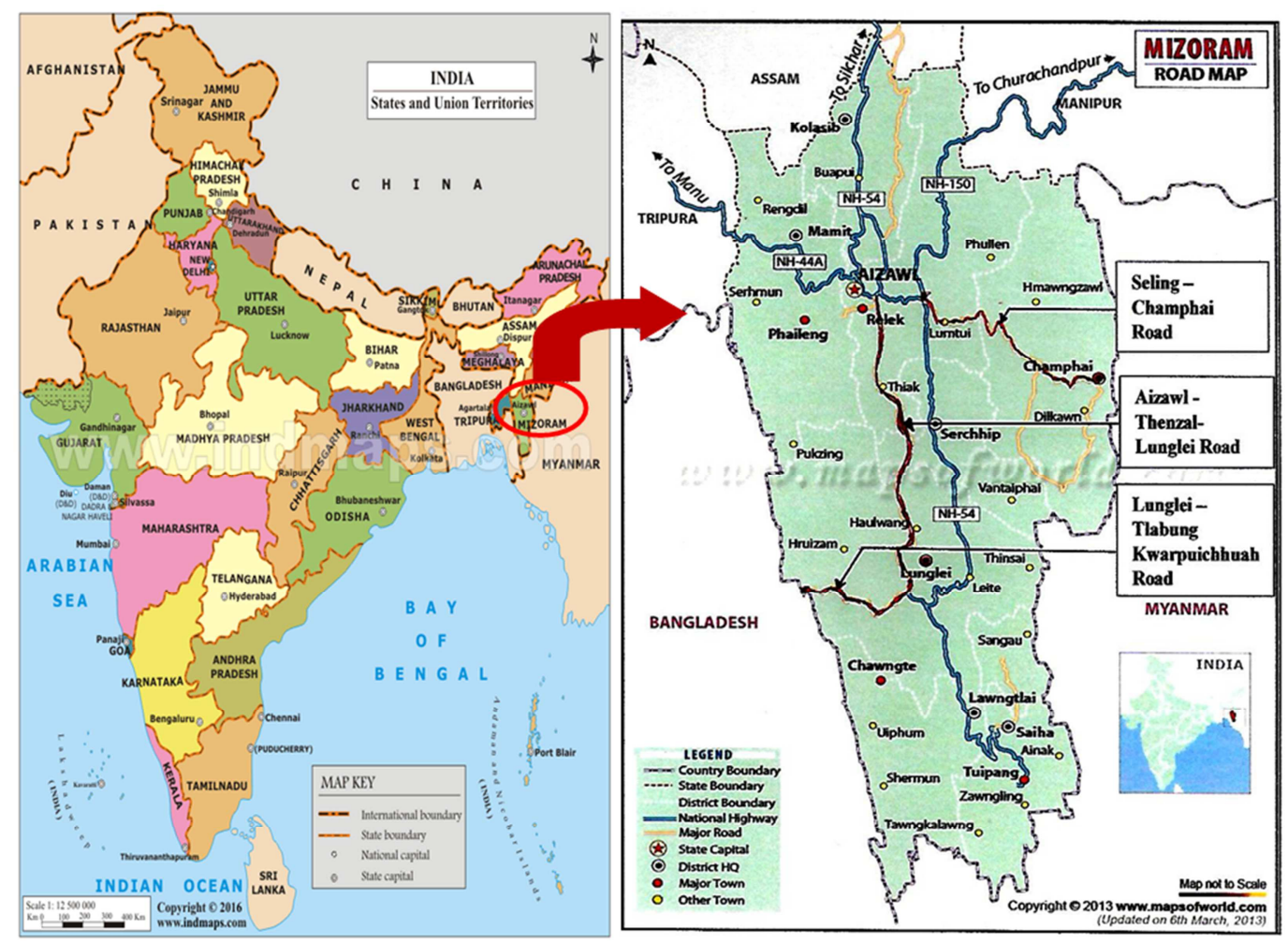

Figure 1. Roads considered for study. 


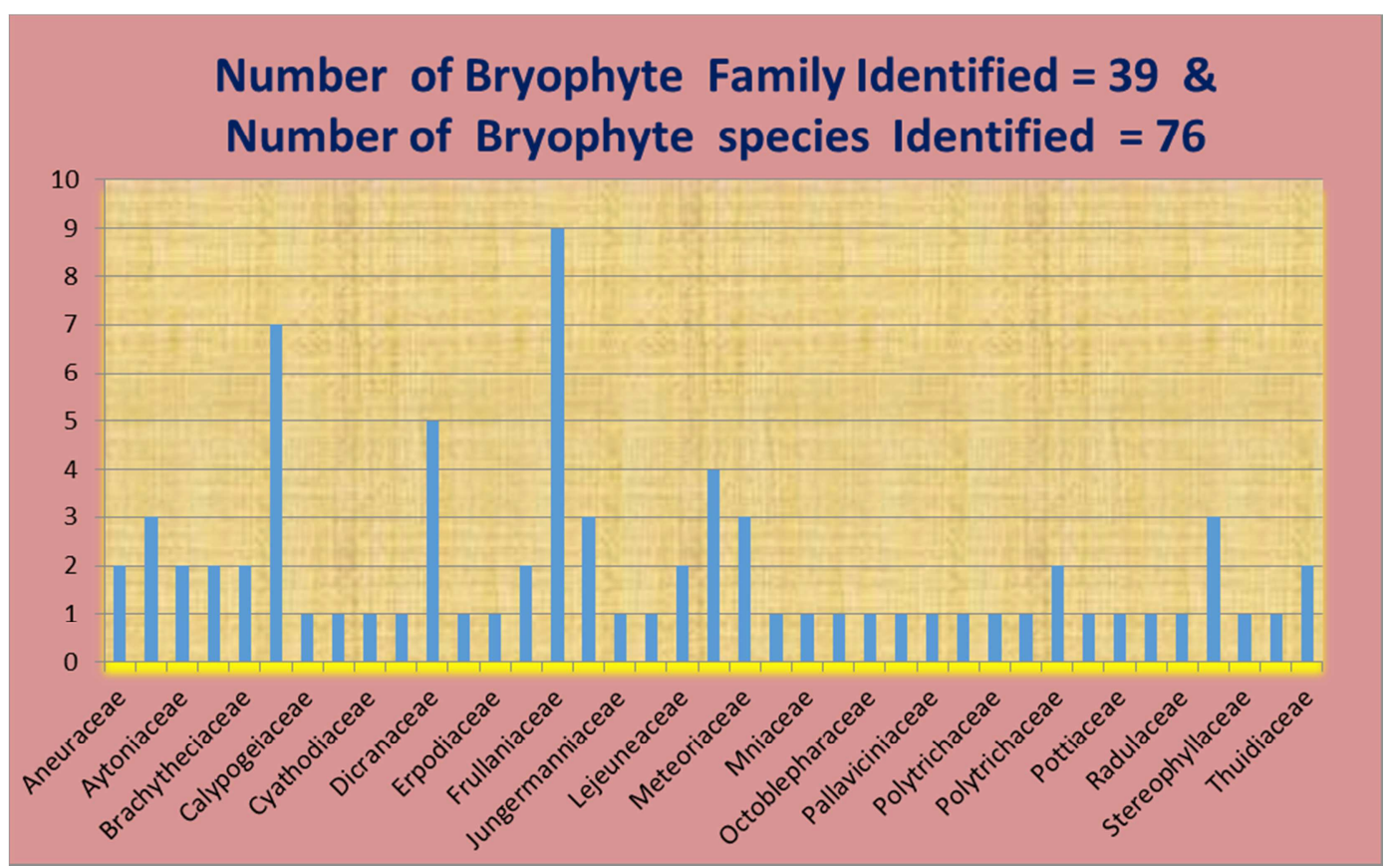

Figure 2. Distribution of Bryophytes along some roads in Mizoram.

Table 2. Traditional use of Bryophytes identified along hill roads.

\begin{tabular}{ll}
\hline Name of the Bryophyte species & Use by local people \\
\hline Marchantia spp & Treatment of Pulmonary tuberculosis and affliction of liver \\
Sphagnum (Decoction) & Acute hemorrhage and diseases of eye \\
Polytrichum commune & Stone of kidney and gall bladder \\
Sphagnum leaves and extracts of Conocephalum conicum, & As Antiseptic for healing of wounds \\
Dumortiera, Sphagnum protoricense, Sphagnum strictum & Bog succession. Use for protection of banks of lakes or shallow bodies of water into solid \\
Sphagnum & soil which supports vegetation \\
Sphagnum & Sphagnum peat is used as fuel, improvement of the soil texture and in surgical dressings \\
Mosses & Used as food for chicks \& birds \\
Mosses & For prevention of soil erosion. They usually grow densely and hence act as soil binders. \\
Mosses & Mosses grow in dense strands forming mat or carpet like structure. \\
Dried mosses and other Bryophytes & Land reclamation., in nurseries beds are covered with thalli of Bryophytes \\
\hline
\end{tabular}

\section{Conclusion}

Bryophytes constitute an important element of diverse vegetation complex which play an important role in terrestrial ecosystems such as modification of habitat, nutrient cycling and the maintenance of nutrient status of the soil, primary production etc. Bryophytes are also potential sources of new antibiotics and anti-cancerous substances. The recent upsurge of interest in monitoring atmospheric pollution has revealed a new aspect of the relevance of this elegant group of plant kingdom. They have been more rationally used in pollution detection, environmental monitoring and as climatic indicator which can be helpful to management of a particular environment.

Due to rapid road development and pressures inflicted by growth of human population and their intense activities influencing the biodiversity of the Bryophytes. If their habitat (s) once disturbed they will certainly be dwindled in their both diversity and abundance. The changes in the microhabitat of bryophytes may seriously affect the species composition very rapidly and thus upset the ecological balance. The conservation of bryophyte is very important in view of their critical role in ecosystem dynamics.

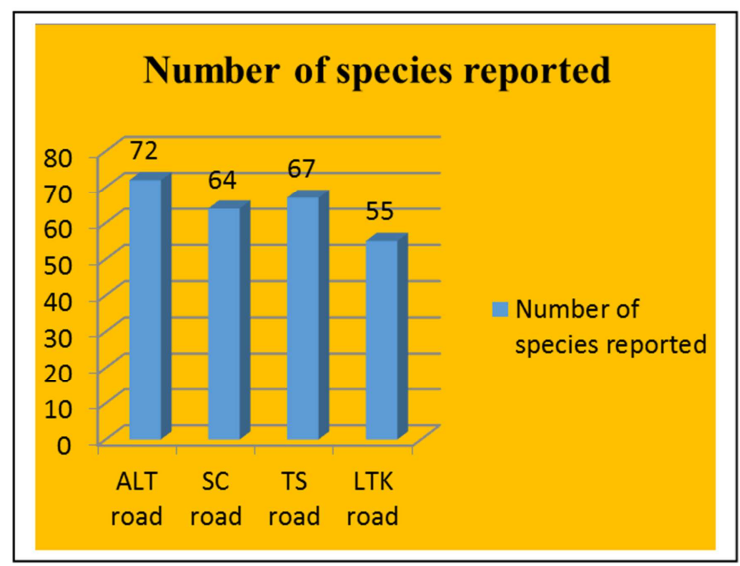

Figure 3. Species wise distribution of Bryophytes along the roads. 


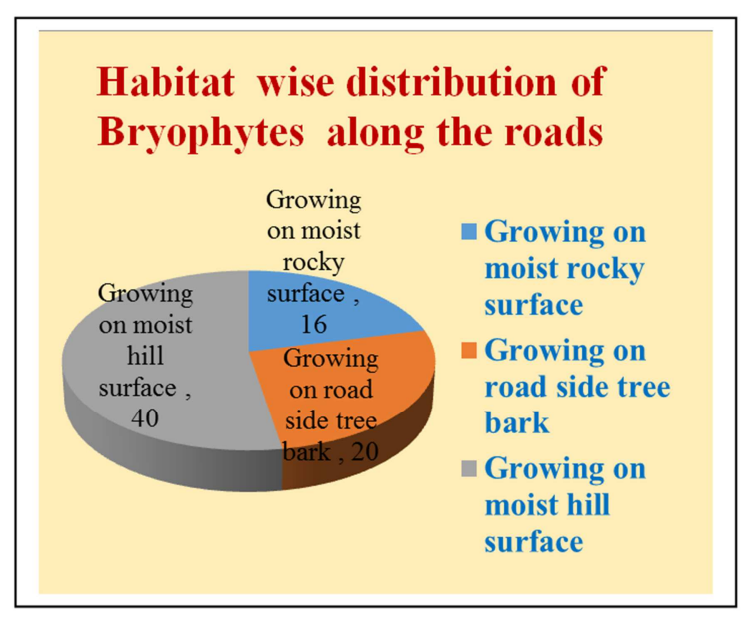

Figure 4. Habitat wise distribution of Bryophytes along the roads.

\section{Acknowledgements}

We are grateful to the Head, Department of Botany Ranchi University, Ranchi, and Professor Ambarish Mukherjee, Department of Botany, Burdwan University, Burdwan for providing facilities guidance and encouragement to complete the work.

\section{References}

[1] Renzaglia, Karen \& Schuette, Scott \& Duff, Robert \& Ligrone, Roberto \& Shaw, Arthur \& D. Mishler, Brent \& Duckett, Jeffrey. (2009). Bryophyte phylogeny: Advancing the molecular and morphological frontiers. The Bryologist. 110. 179-213.

[2] Rubinstein CV, Gerrienne P, Puente G. S. de la, . Astini R. A, Steemans P. (2010). Early Middle Ordovician evidence for land plants in Argentina (eastern Gondwana). New Phytologist (2010) 188: 365-369.

[3] Srivastava, S. C 1998. Distribution of Hepaticae and Anthocerotae in India. In: R. N. Chopra (ed.) Topics in Bryology. Allied Publishers Limited, New Delhi.

[4] Bryophytes in India - list of families and genera of bryophytes in India. ENVIS Centre on Floral Diversity, Botanical Survey of India in 2016.

[5] Chopra R N and Vashistha B D (1994). Bryophyte Morphogenetic Study, In: Botany of India (History and Progress), I edited by Johri BM (Oxford IBH Publishing Co. Pvt. Ltd., New Delhi) 437-453.

[6] Gerson U. (1982), Bryophytes and Invertebrates. In: Smith A. J. E. (eds) Bryophyte Ecology. Springer, Dordrecht.

[7] Chakrabortty, S. and Paratkar, G. T. (2006). Biomonitoring of Trace Element Air Pollution Using Mosses, Aerosol and Air Quality Research, Vol. 6, No. 3, pp. 247-258, 2006.

[8] H. DeLucia, Evan \& Turnbull, Matthew \& S. Walcroft, Adrian \& Griffin, Kevin \& Tissue, David \& Glenny, David \& M. McSeveny, Tony \& Whitehead, David. (2003). The contribution of bryophytes to the carbon exchange for a temperate rainforest, Global Change Biology 9 (8): 11581170 .
[9] Konrat, Matt \& Shaw, Arthur \& Renzaglia, Karen. (2014). A special issue of Phytotaxa dedicated to Bryophytes: The closest living relatives of early land plants. Phytotaxa. 9: 5 10 .

[10] H. Brown, Dennis \& W. Bates, Jeffrey. (2008). Bryophytes and nutrient cycling. Botanical Journal of the Linnean society. 104. 129-147.

[11] Song L, Zhang YJ, Chen X, Li S, Lu HZ, Wu CS, Tan ZH, Liu WY, Shi XM.(2015) Water relations and gas exchange of fan bryophytes and their adaptations to microhabitats in an Asian subtropical montane cloud forest. J Plant Res. 2015 Jul; 128 (4): 573-84.

[12] Dennis Gignac, L. (2009). Bryophytes as Indicators of Climate Change. The Bryologist. 104. 410-420.

[13] Christopher Ellis (2015), Implication of climate change for UK bryophytes and lichens, Biodiversity Climate change impacts reports card technical paper, Royal Botanic Garden Edinburgh.

[14] Vohra, J. N. And M. N. Aziz. 1997. Mosses. In: Mudgal, V. \& R. K. Hajra (Ed.), Floristic Diversity and Conservation Strategies in India 1: 301-374. BSI, Kolkata.

[15] Singh AP., Nath V. Hepaticae of Khasi and Jaintia Hills: Esatern Himalayas. Bishen Singh Mahendra Pal Singh, Dehradun, India, (2007).

[16] Lalhriatpuia and Ramachandra Laha (2015), Bryophyte Diversity In Mamit District, Mizoram, Northeast India, Int J Pharm Bio Sci 2015 Oct; 6 (4): (B) 1204-1209.

[17] Jayanta Barukial (2011) A study of moss diversity in Assam Valley wet evergreen forests. Indian Journal of Fundamental and Applied Life Sciences ISSN: 2231-6345 (Online) Vol. 1 (4): $1-8$

[18] Sudhanshu Kumar Jain, R. Raghavendra Rao, A Handbook of Field and Herbarium Methods, Today \& Tomorrow's Printers and Publishers, 1977.

[19] Gangulee, H. C. 1969-1980. Mosses of Eastern India and adjacent regions. Fasc. 1-8. 1-2142. Calcutta.

[20] Bir, S. S. \& Chopra, R. N. (1972): Thallose Liverworts From Dalhousie, North Western Himalayas. The Bryologist 75: 371372 .

[21] Bansal P and Nath V, Bryum Bessonii Ren. \& Card. New To Eastern Himalaya In New National and Regional Bryophyte Records 32. J. Bryol. 34: 231-246 (2012b).

[22] Bapna, K. R. \& Kachroo, P. (2000): Hepaticology In India Ii. Himanshu Publication, Delhi.

[23] Srivastava, A. \& Srivastava, S. C. (2002): Indian Geocalycaceae (Hepaticae) - a taxonomic study. Bishen Singh Mahendra Pal Singh, Dehradun.

[24] Singh, A. P., Nath, V. and Asthana, A. K. 2002. Studies on Iieteroscyphus perfoliatus (Mont.) Schiffii. from Meghalaya (India). J. Indian Bot. Soc. 81: 305-307.

[25] Plant Discoveries 2016-New Genera, Species and New Records' compiled and edited by Paramjit Singh and S. S. Dash, published by Botanical Survey of India in 2017. 